\title{
Kerion Celsi durch Microsporum canis - erfolgreiche Behandlung mit Terbinafin bei einem Kleinkind
}

\section{Kerion Celsi Due to Microsporum Canis - Successful Treatment with Terbinafine in an Infant}

Autoren

Institute

\author{
A. Laumanns ${ }^{1}$, I. Schulze ${ }^{1}$, P. Nenoff ${ }^{2}$
}

MVZ Haut- und Geschlechtskrankheiten, Markkleeberg

Laboratorium für medizinische Mikrobiologie, Mölbis

\section{Bibliografie}

DOI $10.1055 / \mathrm{s}-2006-944836$ Akt Dermatol 2006; 32; 481-483 @ Georg Thieme Verlag KG Stuttgart · New York ISSN 0340-2541

Korrespondenzadresse

Astrid Laumanns

MVZ Haut- und Geschlechtskrankheiten (Ina Schulze) \& Urologie (Dr. Matthias Schulze) Hauptstraße 10 04416 Markkleeberg A.Laumanns@praxis-schulze.de

\section{Zusammenfassung \\ $\nabla$}

Ein $1 \frac{1}{2}$-jähriges Mädchen litt an einer Tinea capitis profunda, die 10-jährige Schwester an einer Tinea corporis durch Microsporum canis. Neben der lokalen antimykotischen Therapie kam für

\section{Einleitung \\ $\nabla$}

Die Tinea capitis ist eine Infektion des Capillitiums durch bestimmte Dermatophytenarten, die zu den Genus Trichophyton (T.) sowie Microsporum $(M$.) gehören. Betroffen sind am häufigsten Kinder, Erwachsene dagegen selten [1]. Als Erreger werden in Deutschland nach einer Erhebung von 1998 am häufigsten M. canis, T. mentagrophytes und T. verrucosum identifiziert [2]. Nach einer weiteren Studie von Aly et al. [3] ist die Inzidenz zoophiler Arten drastisch gestiegen.

Klinisch unterteilt man die Tinea capitis entsprechend ihrer Ausprägung in drei Formen. Als sog. aphlegmasische Form imponiert sie mit kleinen pityriasiform schuppenden Herden und abgebrochenen Haaren ohne nennenswerte Entzündung. Darüber hinaus kann sich das Krankheitsbild mitunter sogar wie eine Alopecia areata äußern. Erreger sind dann meist Microsporum-Arten, wie M. audouinii und andere anthropophile Arten. Die phlegmasische Form (chronisch-entzündlich und infiltrativ) und die akut infiltrative Form (Maximalvariante Kerion Celsi), bei denen es zu einer starken Entzündungsreaktion mit Pustelbildung und teilweisem Haarverlust, zu Fieber mit Lymphknotenschwellungen und Beeinträchtigung des Allgemeinbefindens kommt, sind eigentlich immer durch zoophile Arten bedingt $[1,2]$.

Eine durch $M$. canis hervorgerufene Tinea capitis (frühere Bezeichnung Mikrosporie) äußert sich durch eine stärkere Entzündungsreaktion [1,2]. Sie ist hochkontagiös und wird fast immer durch das Kleinkind Terbinafin per os im Rahmen eines individuellen Heilversuchs zur Anwendung. Die Verträglichkeit dieses Antimykotikums war ausgezeichnet. Eine nur 4-wöchige Behandlungsdauer des Kerion Celsi reichte aus, um eine Restitutio ad integrum zu erzielen.

Katzen übertragen; das kann in Deutschland sein, viel häufiger jedoch in mittelmeernahen Gebieten. Andere felltragende Tiere, z. B. Hunde, sind nur selten Carrier und Überträger von $M$. canis. Dabei müssen die Tiere nicht offensichtlich befallen sein, sie sind meist nur asymptomatische Träger mit unauffälligem Fell [4]. Möglich ist auch eine Übertragung von Mensch zu Mensch [5].

\section{Patientenbeschreibung \\ $\nabla$}

\section{Anamnese}

Ein $1 \frac{1}{2}$-jähriges Mädchen wurde in der Hautarztpraxis mit einer etwa $5 \mathrm{~cm}$ durchmessenden, fest anhaftenden, gelben Kruste am Oberkopf vorgestellt. Es bestanden keinerlei Vorerkrankungen, Medikamente nahm das Kleinkind nicht ein. Als Haustiere hielt die Familie zwei Meerschweinchen. Gleichzeitig stellte die Mutter des Kindes die 10-jährige Schwester vor, welche am rechten Oberarm eine gerötete, schuppende und zentrafugal wachsende Läsion aufwies. Auch dieses Kind hatte keinerlei Vorerkrankungen oder nahm Medikamente ein. Es bestand ebenfalls Kontakt zu den Meerschweinchen.

\section{Hautbefund}

Parietal imponierte bei dem $1 \frac{1}{2} / 2$-jährigen Mädchen ein ca. $5 \mathrm{~cm}$ im Durchmesser betragendes, kreisrundes und entzündlich erythematöses Infiltrat mit fest haftender gelblich-bräunlicher Kruste $(\bullet$ Abb. 1). Die Haare waren zum Teil ab- 


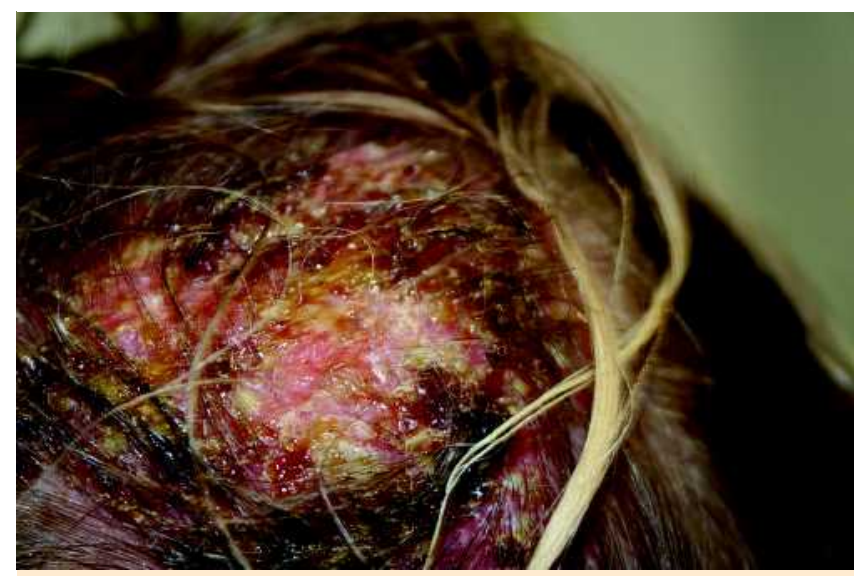

Abb. 1 Tinea capitis profunda bei einem 11/2-jährigen Mädchen: ca. $5 \mathrm{~cm}$ im Durchmesser betragendes, kreisrundes und entzündlich erythematöses Infiltrat mit fest haftender gelblich-bräunlicher Kruste.

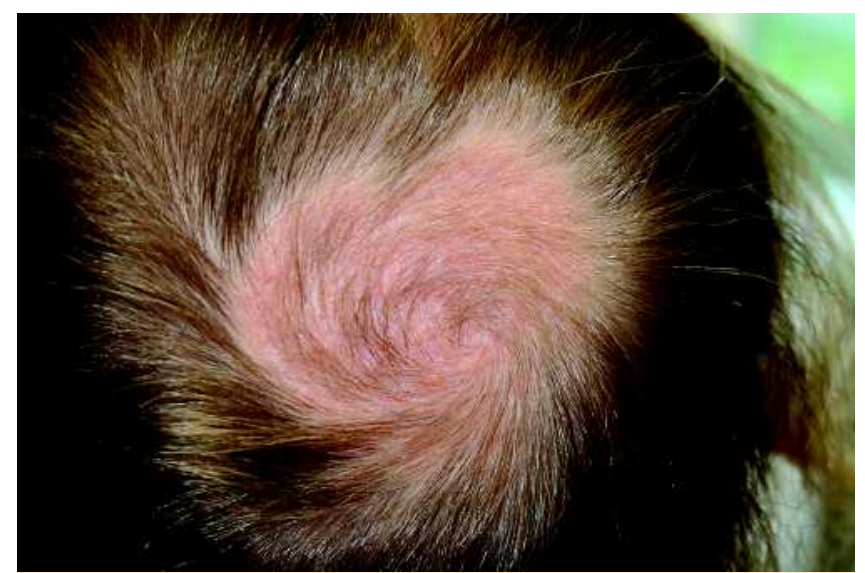

Abb. 3 Tinea capitis profunda nach 4-wöchiger Behandlung mit Terbinafin per os und lokaler antimykotischer Behandlung.

gebrochen oder auch ausgefallen. Es bestand diskreter Pruritus. Am rechten Oberarm der 10-jährigen Schwester des Kleinkindes war ein ca. 3,5 $\times 4 \mathrm{~cm}$ großer, erythematöser und leicht schuppender Herd sichtbar, der klinisch am ehesten einem nummulären Herd eines mikrobiellen Ekzems entsprach. Es bestand auch hier Pruritus.

\section{Diagnostik}

Unter dem Verdacht auf eine Tinea capitis sind Kopfschuppen entnommen worden. Mittels Fluoreszenzmikroskopie (Calcoflor $^{\circledR}$ ) ließen sich Pilzmyzel und -sporen massenhaft nachweisen. Kulturell wurde auf Sabouraud 4\%-Glukose-Agar mit und ohne Cycloheximid (Actidion $^{\circledR}$ ) der zoophile Dermatophyt Microsporum (M.) canis angezüchtet: Die Kolonien der Primärkultur auf Sabouraud-Glukose-Agar/Schrägagarröhrchen waren weiß, flach, ausstrahlend, von der Unterseite der Kolonien schimmerte gelbes Pigment durch ( $\bullet$ Abb. 2). Die Kolonieunterseite war zentral leuchtend gelb bis dunkelgelb, peripher war der Gelbton heller und „wärmer“ (organgegelb). Im Lactophenol-Baumwollblau-Quetschpräparat von der Primärkultur sah man dünnes und verzweigtes, septiertes Myzel, teilweise gelb gefärbt, außerdem zahlreiche Makrokonidien. Diese wiesen eine charakteristische Spindelform auf, zwischen 4 und 8 Kam-

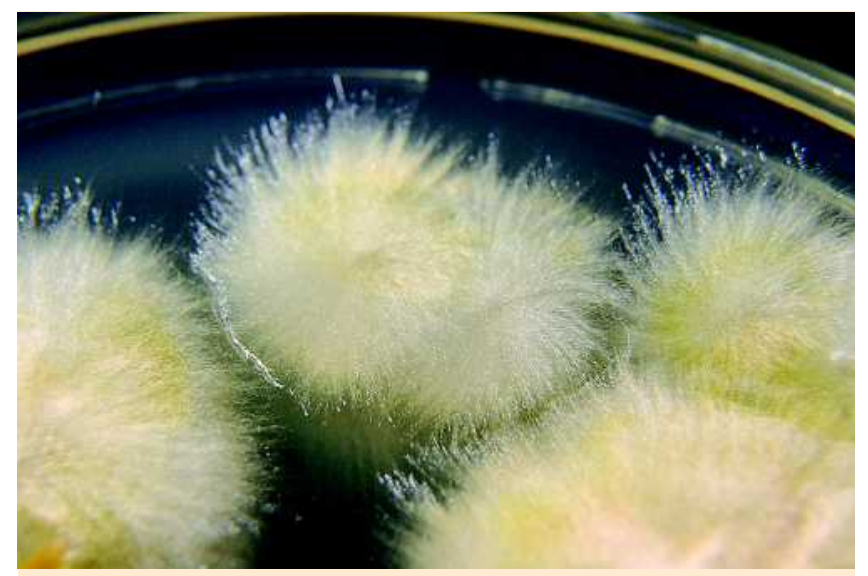

Abb. 2 Microsporum canis-Kolonien. 10 Tage alte Subkultur auf Sabouraud-4\%-Glukose-Nährboden.

mern waren erkennbar. Die Makrokonidien waren auffällig dickwandig, spitz zulaufend an den Polen, im Einzelfall verformt/deformiert (gebogen), dazu kamen zahlreiche runde Mikrokonidien. Aus Hautschuppen der Läsion am Arm der 10-jährigen Schwester war ebenfalls $M$. canis gewachsen. So konnte die Diagnose Tinea capitis et corporis durch $M$. canis gestellt werden. Des Weiteren wurden aufgrund des naheliegenden Übertragungsweges Haare der beiden Meerschweinchen auf Dermatophyten untersucht. Dabei ließen sich lediglich Schimmelpilzspezies (Penicillium spp., Aspergillus flavus, Alternaria spp., Mucor spp.) nachweisen. Die Meerschweinchen wurden zusätzlich von einem Veterinärmediziner untersucht und für klinisch unauffällig bezüglich einer Pilzerkrankung beurteilt.

\section{Therapie und Verlauf \\ $\nabla$}

Initial kam 2\%iges Salicylöl und Ciclopiroxolamin-haltige Creme (Batrafen ${ }^{\circledR}$ Creme) sowie Shampoo (Batrafen ${ }^{\circledR}$ S Shampoo) zur lokalen Behandlung der Kopfhautläsion der jüngeren Patientin zur Anwendung. Nach Vorliegen des Ergebnisses der mykologischen Untersuchung wurde mit $62,5 \mathrm{mg} / \mathrm{d}$ Terbinafin per os (Terbinafin HEXAL ${ }^{\circledR}$ Tabletten, Dosis angepasst an das Körpergewicht) im Rahmen eines individuellen Heilversuchs über 4 Wochen behandelt. Dem zuvor ging eine ausführliche Aufklärung der Eltern, die ihre Einwilligung zu dieser in Deutschland für Kinder nicht zugelassenen systemischen antimykotischen Behandlung erklärten. Klinisch kam es zur vollständigen Heilung der Tinea capitis profunda ( $\odot$ Abb. 3 ). Eine abschließende Untersuchung von Kopfschuppen und Haarwurzeln sprach zusätzlich, bei negativem Fluoreszenzpräparat und keinerlei Erregerwachstum, für eine mykologische Heilung. Die Behandlung wurde, ohne dass Nebenwirkungen auftraten, abgeschlossen.

Das ältere Geschwisterkind wurde zunächst aufgrund des klinischen Bildes mit einer Fusidinsäure- und Betamethasonvalerathaltigen Creme (Fucicort ${ }^{\circledR}$ Creme) lokal behandelt, worunter es bereits zu einer deutlichen Verbesserung kam. Nach Erhalt des mykologischen Untersuchungsergebnisses wurde die Lokaltherapie ebenfalls auf eine Ciclopiroxolamin-haltige Creme (Batrafen ${ }^{\circledR}$ Creme) umgestellt. Der Herd heilte vollständig. 


\section{Besprechung}

$\nabla$

Die Klinik eines entzündlichen, mit Krusten bedeckten alopezischen Herdes am Capillitium eines Kleinkindes sollte neben einer Impetigo contagiosa auch an eine Tinea capitis denken lassen. In diesem Alter werden fungistatisch wirkende gesättigte langkettige Fettsäuren im Sebum noch nicht in dem Maße gebildet, wie dieses ab dem Beginn der Pubertät der Fall ist [5].

Im Schuppenmaterial vom Kopf ließen sich im Fluoreszenzpräparat mit Calcoflor ${ }^{\circledR}$ Pilzelemente nachweisen, kulturell gelang die Anzüchtung von $M$. canis. Daher war es zunächst naheliegend, die beiden im Haushalt lebenden Meerschweinchen veterinärmedizinisch und mykologisch untersuchen zu lassen. Bei Nagetieren, also auch Meerschweinchen, ist selbstverständlich an erster Stelle nicht an M. canis, sondern T. mentagrophytes (aktuelle Nomenklatur: zoophile Varietät von T. interdigitale) zu denken. Weder der Tierarzt noch die mykologische Untersuchung von Haaren der Meerschweinchen konnten jedoch den Nachweis einer Dermatophyten-Infektion bzw. eines Trägerstatus erbringen. Es sei darauf verwiesen, dass hierbei immer die mykologische Untersuchung beweisend ist, nicht zuletzt deshalb, weil ein adäquates Krankheitsbild bei den Tieren oft fehlt und diese lediglich Carrier für die Dermatophyten darstellen [4]. Als Infektionsquelle muss daher ein außerhalb des Haushalts lebendes Tier, beispielsweise eine streunende Katze, angesehen werden.

Als Therapie für die Tinea capitis ist neben der sofortigen lokalen Behandlung mit einem möglichst sporoziden Wirkstoff (Ciclopiroxolamin) eine systemische Therapie nach Erregernachweis notwendig. Bei Kindern ist für die systemische Therapie der Tinea capitis in Deutschland zurzeit nur Griseofulvin uneingeschränkt zugelassen, bei fehlender Alternative kann auch Fluconazol bei Kindern über $1 \mathrm{Jahr}$ verordnet werden [6]. Neuere Medikamente, wie z.B. Terbinafin können eingesetzt werden, um kürzere Behandlungszeiten und damit eine bessere Compliance bei Kindern zu erzielen.

Terbinafin hat als Allylaminantimykotikum in vitro eine exzellente Aktivität gegen alle Dermatophyten. Die minimalen Hemmkonzentrationen liegen bei $0,006 \mu \mathrm{g} \mathrm{ml}^{-1}$ [7]. Prinzipiell gilt, dass Terbinafin für Kinder in Deutschland nicht zugelassen ist - jedoch in der Schweiz, Italien und in Österreich ab dem 2. Lebensjahr -, es gibt dessen ungeachtet jedoch vielfältige Erfahrungen sowohl aus einer Reihe von Studien, als auch individuellen Anwendungen. Entsprechend Arzneimittelgesetz ist ein Einsatz als individueller Heilversuch möglich, mit dem schriftlichen Einverständnis der Eltern [8]. Terbinafin wird abhängig vom Körpergewicht dosiert. Bei Tinea capitis, verursacht durch Trichophyton-Spezies, reicht eine 2-wöchige Therapiedauer [9], wohingegen bei Microsporum-Infektionen - trotz der fungiziden Wirkung - mindestens vier Wochen, im Einzelfall jedoch bis zu 12 Wochen zu behandeln ist [10].

Als zusätzlicher günstiger Nebeneffekt muss die Kosteneinsparung durch kürzere Behandlungszeiten genannt werden. Die Dosierung wird dem Körpergewicht angepasst, so dass Nebenwirkungen selten sind [11,12].

Um die Wirksamkeit und Verträglichkeit von Terbinafin bei der Tinea capitis durch $M$. canis zu untersuchen, behandelten Aste und Pau 26 Patienten - 22 Kinder und vier Frauen - über einen Zeitraum von 12 Wochen [13]. Die angepasste Dosis betrug $62,5 \mathrm{mg} \mathrm{die}^{-1}$ bei Patienten mit einem Körpergewicht $<20 \mathrm{~kg}$, $125 \mathrm{mg} \mathrm{die}^{-1}$ bei denen mit einem Gewicht zwischen 20 und
$40 \mathrm{~kg}$, und $250 \mathrm{mg} \mathrm{die} \mathrm{d}^{-1}$ bei Patienten, die mehr als $40 \mathrm{~kg}$ wogen. 22 Patienten $(84,6 \%)$ waren klinisch und mykologisch geheilt, die Verträglichkeit war exzellent, Nebenwirkungen wurden bei keinem der Patienten beobachtet. Die Paraklinik war bei den Patienten völlig normal.

Das Nebenwirkungsprofil von Terbinafin ist bei Kindern ähnlich dem der Erwachsenen bei gutem Nutzen-Risiko-Verhältnis. Bei der Tinea capitis durch $M$. canis reicht oft bereits eine 4-wöchige Therapie mit Terbinafin gegenüber 8 Wochen Griseofulvin aus [14]. Dem entspricht der Verlauf der Behandlung der Tinea capitis profunda beim hier beschriebenen Kind.

\section{Abstract}

\section{Kerion Celsi Due to Microsporum Canis - Successful Treatment with Terbinafine in an Infant $\nabla$}

A $1 \frac{1}{2}$ years old girl suffered from a tinea capitis profunda, and her 10 years old sister from tinea corporis due to microsporum canis. In addition to a topical antifungal therapy the infant was treated with terbinafine per os as an individual treatment trial. The tolerability of this antifungal substance was excellent. Four weeks treatment duration of the kerion celsi were enough to achieve a restitutio ad integrum.

\section{Literatur}

1 Braun-Falco O, Plewig G, Wolff HH. Dermatologie und Venerologie. Berlin: Springer, 1997: $288-289$

2 Tietz HJ. Tinea capitis - Erregerwandel, Diagnostik und Therapie. Derm Praktische Dermatologie 2005; 11: 10-14

3 Aly R, Hay RJ, Del Palacio A, Galimberti R. Epidemiology of tinea capitis. Med Mycol 2000; 38 (Suppl. 1): 183 - 188

4 Nenoff $P$, Herrmann J. Die Rückkehr der zoophilen Hautpilzinfektionen - Diagnostik und Therapie. ÄP Ärztliche Praxis Dermatologie \& Allergologie 2006; 2: 29-30

5 Nenoff P, Gebauer S, Rytter M, Haustein U-F. Tinea capitis et corporis durch Microsporum canis. Derm Prakt Dermatologie 1997; 3: $302-$ 308

6 Tietz H-J, Sterry W. Antimykotika von A-Z. 4. Auflage. Stuttgart: Thieme, 2006: 125 - 126

7 Nenoff P, Wolf T, Reinl P, Haustein U-F. Aktuelle Aspekte zur Diagnostik und Therapie von Dermatomykosen: In vitro-Empfindlichkeit von Malassezia spp. und Dermatophyten. Tagungsband der Deutschen Veterinärmedizinischen Gesellschaft, Fachgruppe „Bakteriologie und Mykologie“. Leipzig, 15. - 17. Juni 2000: Verlag der Deutschen Veterinärmedizinischen Gesellschaft e. V, 2000: 96 - 110

8 Nenoff $P$. Tinea capitis beim Kind - aktuelle Aspekte der Pathogenese, Epidemiologie sowie Therapie. Deutscher Dermatologe 2003; 10: $756-761$

9 Friedlander SF, Aly R, Krafchik B et al. Terbinafine in the treatment of Trichophyton tinea capitis: a randomized, double-blind, parallelgroup, duration-finding study. Pediatrics 2002; 109: 602 - 607

10 Baleviciene G, Ceburkovas O, Maciuliene D. Oral terbinafine for tinea capitis due to Microsporum canis in children. Acta Derm Venereol 2001; 81: 61

11 Mohrenschlager M, Seidl HP, Ring J, Abeck D. Pediatric tinea capitis: recognition and management. Am J Clin Dermatol 2005; 6: 203-213

12 Chan YC, Friedlander SF. New treatments for tinea capitis. Curr Opinion Inf Dis 2004; 17: $97-103$

13 Aste N, Pau M. Tinea capitis caused by Microsporum canis treated with terbinafine. Mycoses 2004; 47: 428-430

14 Wollina U, Vennewald I. Kreisrunder Haarausfall beim Kind: Tinea capitis durch Microsporum canis. Hautnah Dermatologie 2004; 6: $312-313$ 